\title{
Exhaled breath metabolomics as a noninvasive diagnostic tool for acute respiratory distress syndrome
}

\author{
Lieuwe D.J. Bos ${ }^{1,2}$, Hans Weda ${ }^{3}$, Yuanyue Wang ${ }^{3}$, Hugo H. Knobel ${ }^{3}$, \\ Tamara M.E. Nijsen ${ }^{3}$, Teunis J. Vink ${ }^{3}$, Aeilko H. Zwinderman ${ }^{4}$ Peter J. Sterk ${ }^{2}$ and \\ Marcus J. Schultz ${ }^{1}$
}

Affiliations: 'Intensive Care, Academic Medical Center, University of Amsterdam, Amsterdam, The Netherlands. ${ }^{2}$ Dept of Respiratory Medicine, Academic Medical Center, University of Amsterdam, Amsterdam, The Netherlands. ${ }^{3}$ Philips Research, Eindhoven, The Netherlands. ${ }^{4}$ Clinical Epidemiology and Biostatistics, Academic Medical Center, University of Amsterdam, Amsterdam, The Netherlands.

Correspondence: Lieuwe D.J. Bos, Dept of Intensive Care and Respiratory Medicine, Academic Medical Center, Meibergdreef 9, G3-228, 1105 AZ Amsterdam, The Netherlands. E-mail: I.d.bosdamc.uva.nl

ABSTRACT There is a need for biological markers of the acute respiratory distress syndrome (ARDS). Exhaled breath contains hundreds of metabolites in the gas phase, some of which reflect (patho)physiological processes. We aimed to determine the diagnostic accuracy of metabolites in exhaled breath as biomarkers of ARDS.

Breath from ventilated intensive care unit patients $(n=101)$ was analysed using gas chromatography and mass spectrometry during the first day of admission. ARDS was defined by the Berlin definition. Training and temporal validation cohorts were used.

23 patients in the training cohort $(n=53)$ had ARDS. Three breath metabolites, octane, acetaldehyde and 3-methylheptane, could discriminate between ARDS and controls with an area under the receiver operating characteristic curve (AUC) of 0.80 . Temporal external validation (19 ARDS cases in a cohort of 48) resulted in an AUC of 0.78. Discrimination was insensitive to adjustment for severity of disease, a direct or indirect cause of ARDS, comorbidities, or ventilator settings. Combination with the lung injury prediction score increased the AUC to 0.91 and improved net reclassification by 1.17 .

Exhaled breath analysis showed good diagnostic accuracy for ARDS, which was externally validated. These data suggest that exhaled breath analysis could be used for the diagnostic assessment of ARDS.

@ERSpublications

Metabolites in the breath of ventilated patients may be used to diagnose the acute respiratory distress syndrome http://ow.ly/uWHF1

This article has supplementary material available from www.erj.ersjournals.com

Received: Jan 082014 | Accepted after revision: March 172014 | First published online: April 172014

Clinical trial: This study is registered at www.trialregister.nl with identifier number NTR 2750

Support statement: L.D.J. Bos is supported by a research grant (PhD Scholarship) from the Academic Medical Center, by an unrestricted research grant from Philips Research and by the ESICM Young Investigator Award.

Conflict of interest: Disclosures can be found alongside the online version of this article at www.erj.ersjournals.com

Copyright @ERS 2014 


\section{Introduction}

As stated in the updated and improved consensus criteria for acute respiratory distress syndrome (ARDS), a valid and reliable definition of ARDS is considered essential for clinical management and to facilitate enrolment of consistent patient phenotypes into clinical trials [1]. The presently used Berlin criteria are empirically selected clinical, radiological and physiological variables [1]. This definition is highly suitable for epidemiological studies but shows only a moderate correlation with post mortem pathological findings [2], and ARDS can still be mistaken for pneumonia or cardiogenic pulmonary oedema, and vice versa $[2,3]$. Therefore, there is need for molecular markers to group phenotypes more objectively and consistently [4].

A diagnostic molecular marker should improve classification over the pre-test probability of disease $[5,6]$. In the case of ARDS, risk factors and risk modifiers have been thoroughly assessed, externally validated and incorporated into the Lung Injury Prediction Score (LIPS) [7, 8]. Discovery and validation of biological markers that reflect the pathophysiological mechanisms underlying lung injury may allow for improved diagnosis before the clinical definition of ARDS is met [9].

Metabolomics can provide an integrated view of upstream physiological, genomic, transcriptomic and proteomic data by assessment of the composition of metabolite mixtures in biological material [10-12] Exhaled breath contains metabolites that are volatile [13]. Importantly, as opposed to exhaled breath condensate collection, analysis of volatile organic compounds (VOCs) in the gas phase does not rely on analysis of soluble markers. Instead, volatile metabolites can be trapped onto a sorbent tube and detected using gas chromatography and mass spectrometry (GC-MS) [14, 15]. Exhaled VOCs can be of systemic origin, can be produced in the lung (e.g. under influence of oxidative stress and inflammatory response) or can be the result of bacterial metabolism [16-21]. Pre-clinical studies provide evidence that lung injury induces changes in exhaled metabolites [17, 22]. Therefore, it is timely to validate the diagnostic accuracy of exhaled metabolomics in ARDS, by using a training set as well as a validation set of patients and controls [23].

We hypothesised that exhaled breath analysis by GC-MS can accurately diagnose ARDS in ventilated intensive care unit (ICU) patients. We aimed to investigate the accuracy, reproducibility and robustness of this diagnosis and the classification of patients with CPE and pneumonia using exhaled breath analysis. This was done by following international guidelines on validating diagnostic accuracy as provided by the Standards for the Reporting of Diagnostic Accuracy Studies (STARD) initiative (table E1) [24]. Finally, we studied the classification performance for ARDS of exhaled breath analysis when combined with the $a$ priori risk, represented by the LIPS.

\section{Methods}

\section{Ethical approval and informed consent}

The institutional review board (IRB) of the Academic Medical Center, Amsterdam, the Netherlands, decided that the study did not fulfil the criteria for medical research as stated in the Dutch "Law on medical research" because of the noninvasiveness and absence of burden of examining exhaled air (IRB: 10.17.0729). It was judged by the IRB that exhaled breath should be analysed without the informed consent of the patient. This trial was registered at the Dutch Trial Register (identifier NTR 2750, www.trialregister.nl).

\section{Design, subjects and setting}

This was a prospective, single-centre cohort study. All patients admitted to the ICU, with the exception of cardiopulmonary surgery patients, were screened between December 2011 and April 2013. The only inclusion criterion was mechanical ventilation within the first $24 \mathrm{~h}$ of ICU admission. The only exclusion criterion was previous ICU admission or mechanical ventilation. Patients who were included before June 2012 entered the training cohort and the remaining patients were used for temporal external validation [25]. Patients were categorized into four groups: controls, ARDS, pneumonia without ARDS and cardiogenic pulmonary oedema.

\section{Clinical diagnosis of ARDS}

A team of trained clinical research fellows prospectively scored the presence of ARDS [26], which was later re-evaluated according to the new Berlin definition [1]. All observers were trained on several occasions before the start of the study. All assessors had attended meetings in which clinical case vignettes were discussed and had $\geqslant 6$ months of experience [27].

\section{Competing diagnoses}

The diagnosis of community- or hospital-acquired pneumonia consisted of adapted Center for Disease Control criteria and a post hoc likelihood of infection was scored (none, possible, probable or proven) (fig. E1 and table E2) $[27,28]$. In contrast to ARDS, the diagnosis of cardiogenic pulmonary oedema required that the 
findings (acute onset, bilateral infiltrates and arterial oxygen tension $\left(\mathrm{PaO}_{2}\right)$ /inspiratory oxygen fraction $\left(\mathrm{FIO}_{2}\right)$ ratio $<300$ ) were fully explained by cardiac dysfunction based on echocardiography [1].

\section{Exhaled breath analysis}

Exhaled breath was sampled and analysed by a standardised methodology that has been previously published [29]. In short, breath was collected through a disposable side-stream connection for $10 \mathrm{~min}$ and VOCs were stored on a sorbent tube. These tubes were analysed by means of GC-MS.

\section{Statistical analysis}

Differences between the groups were compared using the Mann-Whitney U or Kruskal-Wallis test for continuous variables and Chi-squared for categorical variables. Data are presented as median (interquartile range) for skewed variables, mean $(95 \% \mathrm{CI})$ for normally distributed variables and $\mathrm{n}(\%)$ for categorical variables. All analyses were performed in R statistics using the R-studio interface (www.r-project.org). p-values $<0.05$ were considered significant.

The metabolomic data were analysed using an a priori data analysis plan (fig. 1) that followed the latest recommendations for metabolomics $[30,31]$ and is described per step here and in the results section. The first step was feature selection and training of the algorithm. To select the most relevant features, the training cohort was repeatedly split into a training set (two-thirds of the cohort) and a test set (the remaining third of the cohort). 1000 iterations of sparse partial least squares logistic regression analysis $(\mathrm{K}=3, \eta=0.9)$ were performed. This is a method for penalised feature selection and is a form of regression that can select predictive variables and limit false discovery in situations where large numbers of independent variables are investigated in low numbers of individuals [32]. The five most frequently chosen ion-fragments (number of covariates in a model is number of included patients divided by 10 [33]) were selected. A logistic regression model was fitted using the selected ion-fragments in the training cohort. We will refer to the result of that model as the "exhaled breath signal". The logistic regression model was applied to the complete training cohort and the area under the receiver operating characteristic curve (AUC) with bootstrapped 95\% confidence interval was reported. The second and third steps were to test the
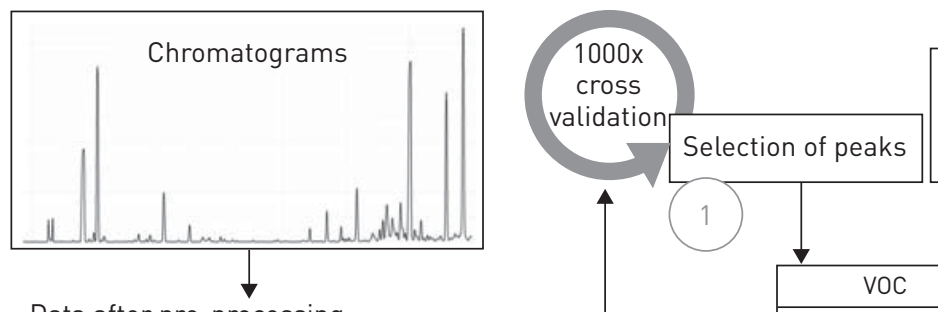

Data after pre-processing

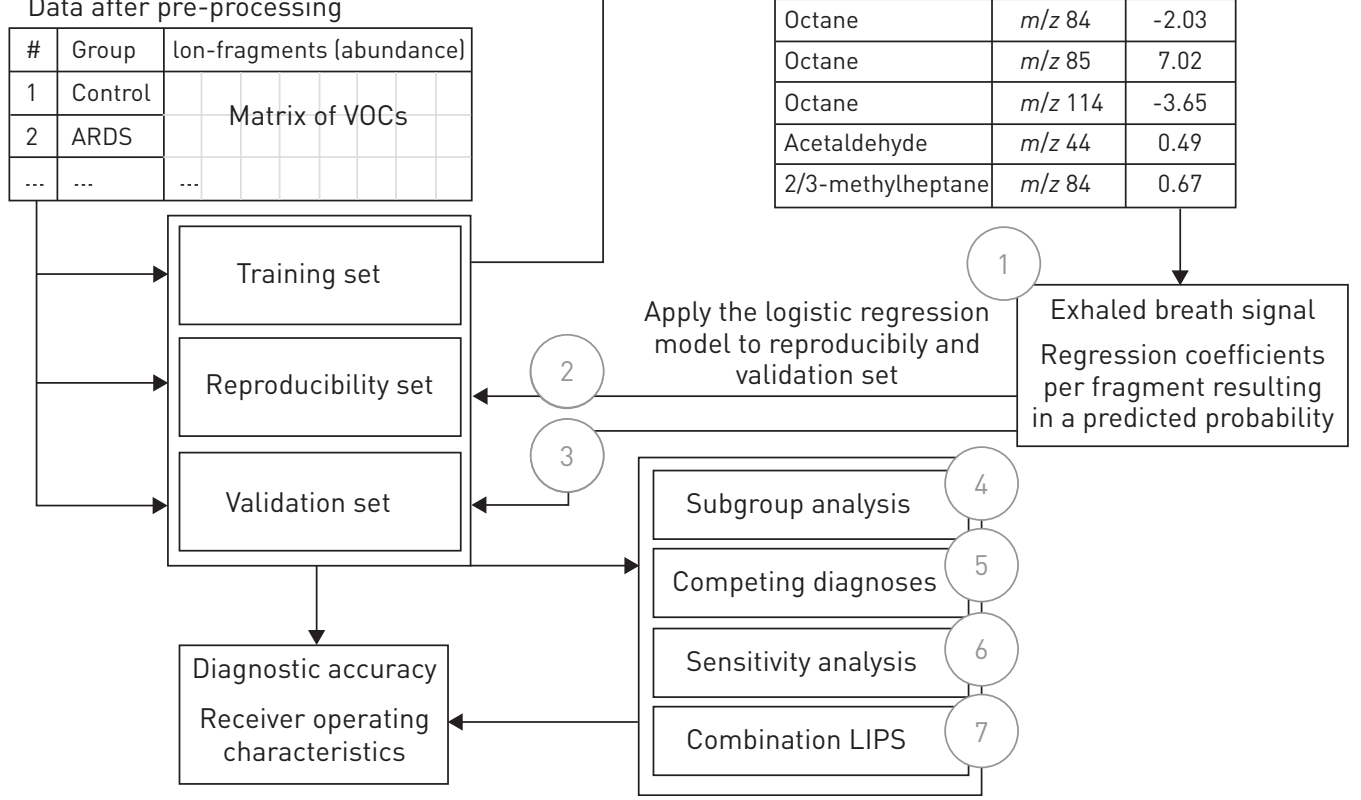

FIGURE 1 Statistical analysis plan. ARDS: acute respiratory distress syndrome; VOC: volatile organic compound; $\beta$ : regression coefficient per selected ion-fragment; $\mathrm{m} / \mathrm{z}$ : mass/charge ratio; LIPS: Lung Injury Prediction Score. 
reproducibility and the temporal external validation by applying the logistic regression model to another breath sample, obtained at the same time-point in the patients of the training cohort, and to a new group of patients, respectively, and compare the AUC. The calibration of the predicted and observed probability of group membership was visualised in a correlation plot (fig. E4). Step four was to perform a subgroup analysis for pulmonary/nonpulmonary and mild/moderate/severe ARDS. Step five was to investigate the diagnostic accuracy of the exhaled breath signal for competing diagnoses. Step six was a sensitivity analysis for comorbidities (chronic respiratory disease, malignancies and diabetes mellitus), bacterial growth in respiratory samples, ventilator settings and measures of severity of disease (Acute Physiology and Chronic Health Evaluation (APACHE) II and Simplified Acute Physiology Score (SAPS) II). Finally, the net reclassification improvement [6] of the combination with LIPS was investigated.

False discovery and over-fitting were avoided by using a temporal external validation cohort [30, 31] with a required sample size of 18 ARDS patients to find $90 \%$ sensitivity with a lower confidence limit of 0.55 (i.e. statistical significance) [34].

\section{Results}

Subjects

300 patients were screened, of whom 140 were not eligible and 59 met the exclusion criterion (fig. 2). Thus, 101 patients were included. No adverse events were reported during or shortly after breath collection. 42 (42\%) patients fulfilled the clinical definition of ARDS [1]: 29 patients were classified as having mild ARDS, and 13 and two patients as moderate and severe ARDS, respectively (table 1). 30 patients had ARDS due to a pulmonary cause (pneumonia $(n=22)$, aspiration $(n=2)$ or other $(n=6))$ and 12 due to a nonpulmonary cause (sepsis $(n=8)$, pancreatitis $(n=1)$ or other $(n=3)) .52(52 \%)$ patients did not fulfil the definition for ARDS; these patients served as control patients. Competing diagnoses were pneumonia (three patients) and cardiogenic pulmonary oedema (four patients). None of the control patients progressed towards ARDS during the first 3 days of ICU admission.

\section{Feature selection and training of algorithm}

23 patients with ARDS and 25 controls were included in the training cohort (table E4 shows baseline characteristics for this cohort). Five ion-fragments generated by the mass spectrometer, which originated from three VOCs (octane (Chemical Abstract Service (CAS) registry number 111-65-9), acetaldehyde (CAS 75-07-0) and 3-methylheptane (CAS 589-81-1)), were selected by the algorithm (fig. 1 and online supplementary material) and were used as predictive variables in a logistic regression model (table E3).

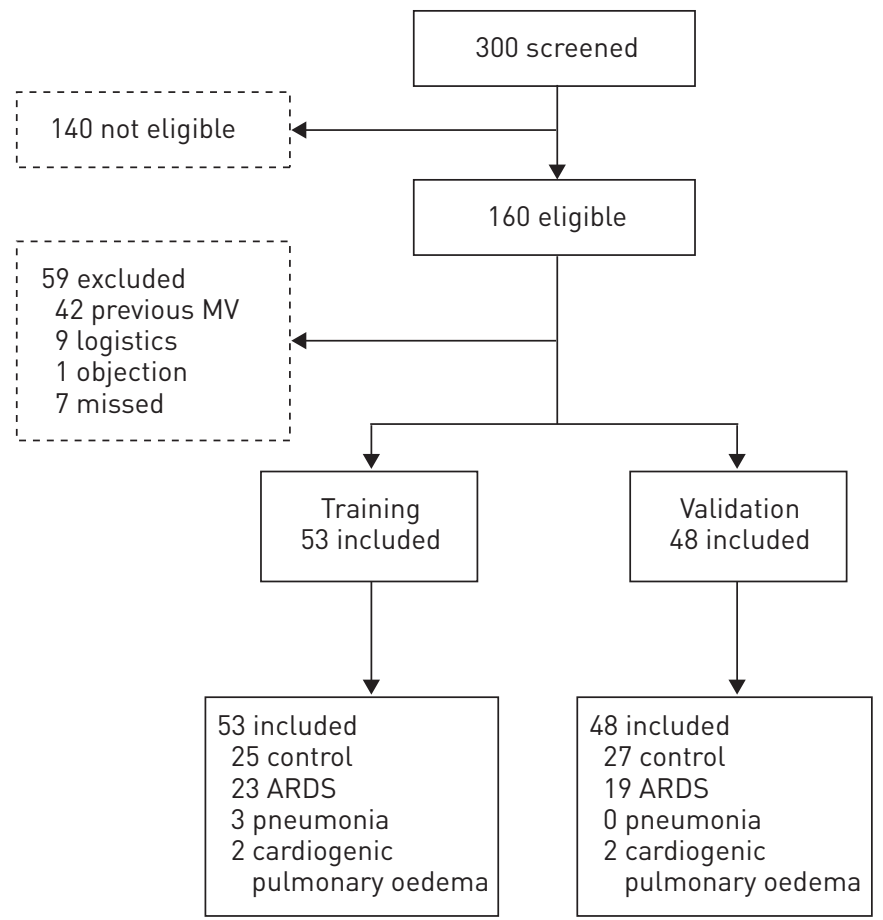

FIGURE 2 Inclusion chart. MV: mechanical ventilation; ARDS: acute respiratory distress syndrome. 
TABLE 1 Patient characteristics: combined training and validation cohort

\begin{tabular}{|c|c|c|c|c|c|}
\hline & Control & ARDS & Pneumonia & $\begin{array}{l}\text { Cardiogenic } \\
\text { pulmonary oedema }\end{array}$ & p-value \\
\hline Subjects $\mathrm{n}$ & 52 & 42 & 3 & 4 & \\
\hline Age years & $60(46-70)$ & $67(48-75)$ & 57 (39-68) & $69(66-71)$ & 0.42 \\
\hline Males & $31(70)$ & $35(67)$ & 1 (33) & $1(25)$ & 0.16 \\
\hline APACHE II & $20(14-26)$ & $23(18-29)$ & $28(19-31)$ & $26(24-30)$ & 0.12 \\
\hline SAPS II & $44(35-64)$ & $46(39-62)$ & $55(41-66)$ & $63(59-71)$ & 0.079 \\
\hline \multicolumn{6}{|l|}{ Admission type } \\
\hline Medical & $34(65)$ & $31(74)$ & $2(67)$ & $2(50)$ & 0.89 \\
\hline Elective surgery & $10(19)$ & $8(19)$ & $1(33)$ & $2(50)$ & \\
\hline Emergency surgery & $3(6)$ & $3(7)$ & $0(0)$ & $0(0)$ & \\
\hline \multicolumn{6}{|l|}{ Comorbidities } \\
\hline Asthma & $0(0)$ & 1 (2) & $0(0)$ & $0(0)$ & 0.49 \\
\hline COPD & $1(2)$ & $2(5)$ & $1(33)$ & $0(0)$ & 0.24 \\
\hline Other respiratory & $1(2)$ & $2(5)$ & $0(0)$ & $0(0)$ & 0.68 \\
\hline Malignancy & $4(8)$ & $7(16)$ & 1 (33) & $2(50)$ & 0.09 \\
\hline DM & $6(12)$ & $9(20)$ & $0(0)$ & $0(0)$ & 0.34 \\
\hline$P \operatorname{Imax} \mathrm{CmH}_{2} \mathrm{O}$ & $16(13-18)$ & $23.5(18-29)$ & 15 (13-19) & 20 (18-22) & $<0.001$ \\
\hline Pplateau $\mathrm{cmH}_{2} \mathrm{O}$ & $14.0(12-18)$ & $22.5(18-28)$ & $14.0(12-18)$ & $18.5(17-21)$ & $<0.001$ \\
\hline PEEP $\mathrm{cmH}_{2} \mathrm{O}$ & $5(5-5)$ & $10(5-10)$ & $5(5-5)$ & $7.5(5-10)$ & $<0.001$ \\
\hline$V T \mathrm{~mL}$ & $450(391-510)$ & $486(397-569)$ & 670 (547-992) & $458(387-540)$ & 0.28 \\
\hline$V_{T} / \mathrm{IBW} \mathrm{mL} \cdot \mathrm{kg}^{-1}$ & $6.5(5.6-7.5)$ & $6.8(6.1-7.6)$ & $11.6(9.7-14.6)$ & $7.3(7.0-8.3)$ & 0.057 \\
\hline Minute volume $L \cdot \min ^{-1}$ & $8.9(7.5-10.9)$ & $10.9(8.7-13.6)$ & $11.1(10.6-12.0)$ & $9.9(9.2-10.9)$ & 0.014 \\
\hline $\mathrm{PaCO}_{2} \mathrm{kPa}$ & $5.1(4.5-5.5)$ & $5.4(4.7-6.0)$ & $4.6(4.3-5.0)$ & $5.2(5.0-5.5)$ & 0.15 \\
\hline $\mathrm{PaO}_{2} \mathrm{kPa}$ & $14.7(13.0-22.2)$ & $12.7(11.0-15.5)$ & $15.7(15.2-18.9)$ & $13.7(13.4-15.0)$ & 0.019 \\
\hline $\mathrm{FlO}_{2} \%$ & $35(30-40)$ & $40(40-56)$ & $40(35-45)$ & $40(39-43)$ & $<0.001$ \\
\hline $\mathrm{PaO}_{2} / \mathrm{FlO}_{2}$ & $360(281-454)$ & 233 (174-297) & $294(257-423)$ & $270(251-286)$ & $<0.001$ \\
\hline LIPS & $2.5(1.5-4.0)$ & $5.0(3.5-5.0)$ & $5.0(3.5-5.0)$ & $3.0(2.5-4.0)$ & $<0.001$ \\
\hline ICU mortality & $5(10)$ & $12(29)$ & $1(33)$ & $1(25)$ & 0.17 \\
\hline \multicolumn{6}{|c|}{$\begin{array}{l}\text { Data are presented as median (interquartile range) or } \mathrm{n}(\%) \text {, unless otherwise stated. Differences between groups were tested using Kruskal- } \\
\text { Wallis one-way ANOVA or Chi-square test (with Yates' correction if necessary). ARDS: acute respiratory distress syndrome; APACHE: Acute } \\
\text { Physiology and Chronic Health Evaluation; SAPS: Simplified Acute Physiology Score; COPD: chronic obstructive pulmonary disease; DM: diabetes } \\
\text { mellitus; PImax: maximal inspiratory pressure; Pplateau: plateau pressure; PEEP: positive end-expiratory pressure; VT: tidal volume; IBW: ideal body } \\
\text { weight derived from height and sex; } \mathrm{PaCO}_{2} \text { : arterial carbon dioxide tension; } \mathrm{PaO}_{2} \text { : arterial oxygen tension; } \mathrm{FlO}_{2} \text { : inspiratory oxygen fraction; LIPS: } \\
\text { Lung Injury Prediction Score; ICU: intensive care unit. }\end{array}$} \\
\hline
\end{tabular}

Figures E2 and E3 show the relative abundance of the fragments between patients with and without ARDS in the training cohort. The diagnostic accuracy of this exhaled breath signal for ARDS in the training cohort was good (AUC 0.80, 95\% CI 0.66-0.92) (fig. 3).

\section{Reproducibility}

The exhaled breath signal derived from the three VOCs was reproducible using another breath sample taken from the same patient population within 10 min of the other sample (AUC 0.78, 95\% CI 0.65-0.91) (fig. 3).

\section{Temporal external validation}

19 patients with ARDS and 27 controls were included in the validation cohort (table E5 shows baseline characteristics for this cohort). The exhaled breath signal from the same three VOCs showed moderate-togood diagnostic accuracy in a new cohort of patients (AUC 0.78, 95\% CI 0.65-0.91) (fig. 3). Figure E4 shows the relative abundance of the five fragments selected by the algorithm between patients with and without ARDS in the temporal external validation cohort.

\section{Subgroup analyses}

The logistic regression function was not different between patients with ARDS due to a pulmonary and a nonpulmonary hit $(\mathrm{p}=0.24)$ (fig. E6). Discrimination of both pulmonary and nonpulmonary ARDS from controls was also not different (AUC 0.75 (95\% CI 0.63-0.86) and 0.80 (95\% CI 0.63-0.96) for pulmonary and nonpulmonary ARDS, respectively; test between AUCs p $=0.63$ [35]).

The exhaled breath signal was not different between patients with mild or moderate/severe ARDS $(p=0.21)$ (fig. E6) and was not correlated with the $\mathrm{PaO}_{2} / \mathrm{FIO}_{2}$ (Spearman's correlation 0.18, $\mathrm{p}=0.27$ ). Discrimination 
FIGURE 3 The diagnostic accuracy of breath analysis for acute respiratory distress syndrome was similar in the training and temporal external validation cohort. AUC: area under the receiver operating characteristic curve.

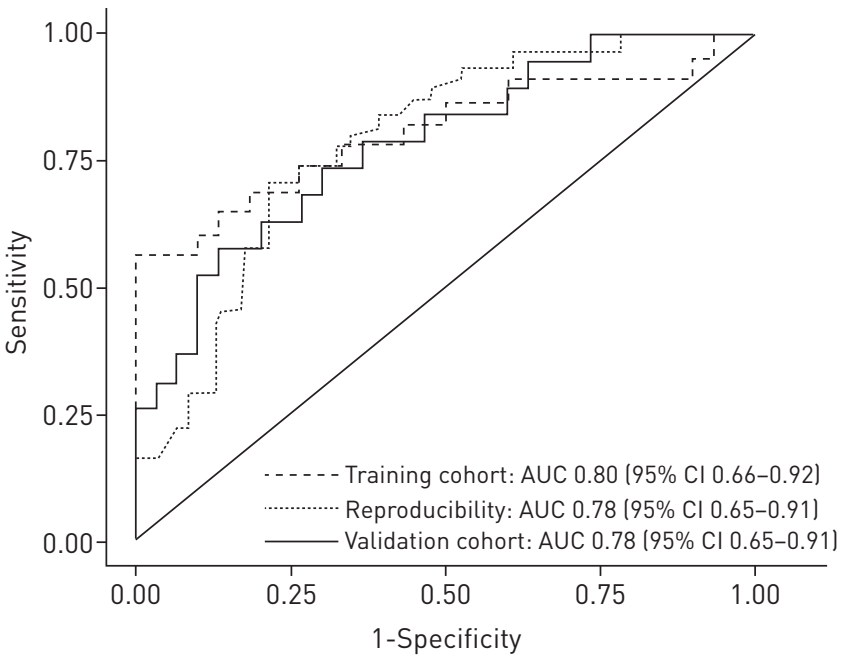

between ARDS and controls was also not different (test between AUCs $p=0.19$ ) between mild (AUC 0.80 , 95\% CI 0.70-0.90) and for moderate/severe ARDS (AUC 0.65, 95\% CI 0.44-0.86).

\section{Competing diagnoses}

Discrimination of cardiopulmonary oedema and pneumonia patients (not having ARDS) from ARDS was excellent (AUC 0.91 (95\% CI 0.80-1.0) and 0.90 (95\% CI 0.81-1.0) for cardiogenic pulmonary oedema and pneumonia, respectively).

\section{Sensitivity analysis}

The influence of covariates on the association between exhaled breath and ARDS was assessed by comparing the log odds ratio of the signal derived from the three VOCs (4.5, 95\% CI 2.6-6.6) for ARDS in an unadjusted logistic regression model to the log odds ratio found in a logistic regression model adjusted for the covariate. The log odds ratio was not sensitive to changes in comorbidities (4.7), bacterial growth in respiratory samples (4.5), maximal inspiratory pressure (4.8), positive end-expiratory pressure (4.9), $\mathrm{FIO}_{2}$ (4.6), $\mathrm{PaO}_{2} / \mathrm{FIO}_{2}$ (5.7), smoking (4.7), alcohol consumption (4.7), APACHE II score (4.3) and SAPS II score (4.2).

\section{Combination with LIPS improves classification}

The LIPS score alone showed moderate/good discrimination between ARDS and controls (AUC 0.78, 95\% CI $0.70-0.87$ ) in the combined training and validation cohort. Discrimination improved when combined with the exhaled breath signal (AUC $0.91,95 \%$ CI $0.85-0.97 ; \mathrm{p}=0.001$ versus LIPS alone, $\mathrm{p}=0.001$ versus exhaled breath signal alone) (fig. 4). The net reclassification improvement was 1.17 (95\% CI 0.79-1.54; $\mathrm{p}<0.001)$.

\section{Discussion}

This study shows that exhaled breath analysis provides good diagnostic accuracy for ARDS in ventilated ICU patients. Discrimination was reproducible and the diagnostic performance was similar after temporal external validation. The exhaled breath diagnosis of ARDS was not influenced by severity of illness, ventilator settings or the examined comorbidities. Furthermore, the diagnostic accuracy increased when the exhaled breath analysis was combined with the LIPS. These findings indicate that exhaled breath analysis qualifies as diagnostic tool for the assessment of ARDS. Clinical application of this technology will be facilitated by further elevation of the diagnostic accuracy.

The described AUC of 0.80 for distinguishing ARDS patients from other ventilated ICU patients is higher than most previously described biological markers, such as interleukins, surfactant proteins and selectins [36]. In contrast to most other studies, we followed an untargeted approach for biomarker discovery. Our data extend previous "omics" approaches using plasma or bronchoalveolar lavage (BAL) in diagnosing ARDS. A recent study that focused on metabolomics reported that the metabolite profile is altered in plasma of patients with sepsis-induced lung injury [37]. Previously, we argued that in the case of ARDS, preferably the lung should be assessed [15]. Indeed, experimentally induced lung injury promotes profound metabolomic changes in BAL and lung tissue $[38,39]$. Taken together, our findings demonstrate that 


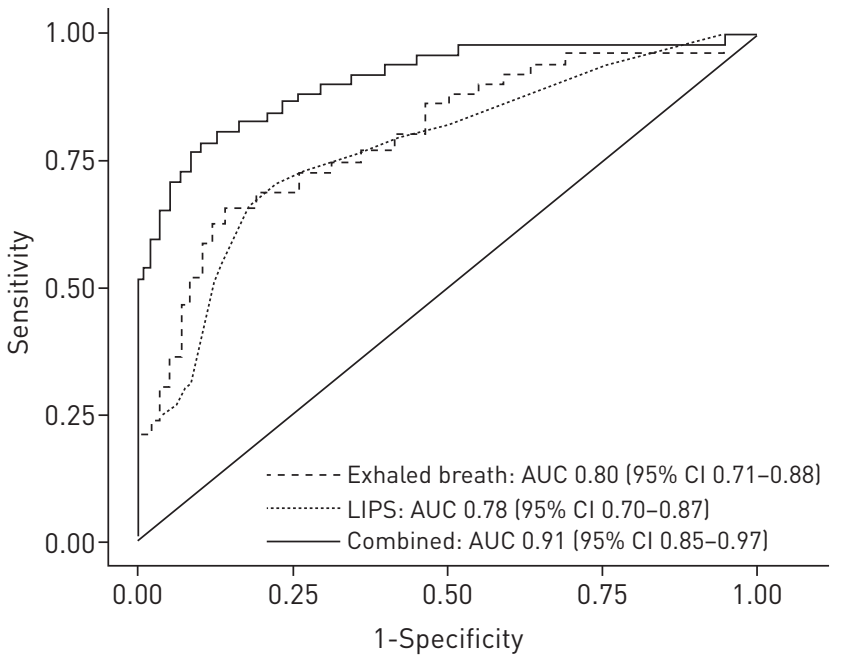

FIGURE 4 The addition of breath analysis to the Lung Injury Prediction Score (LIPS) improved classification. AUC: area under the receiver operating characteristic curve.

metabolomic profiling in ARDS is not only effective in plasma and BAL, but that exhaled breath also provides accurate diagnostic information on the altered metabolites in ARDS.

We identified three volatile biological markers of ARDS: octane, acetaldehyde and 3-methylheptane. However, we observed no differences in exhaled isoprene concentrations between patients with and without ARDS (figure E7), as reported in a pioneering paper by SCHUBERT et al. [19]. The two studies differ on two major points: the conceptual approach and timing of patient selection. Concerning the first, we did not specify what compounds may be associated with ARDS a priori, whereas the previous study was hypothesisdriven by focussing on exhaled acetone, isoprene and pentane. Second, our patients were included within $24 \mathrm{~h}$ of ICU admission, thereby early in the development of ARDS, whereas the patients in the previous study were included later in the course of disease. At the time, isoprene was linked to cholesterol metabolism and neutrophil activity but recent evidence connects isoprene to muscle activity [40]. In ICU patients, muscle activity is affected by ICU-acquired weakness, coma, sedation and delirium. Thus, we could not reproduce the findings of the only previously published study on breath biomarkers of ARDS, which may be due to methodological differences or due to imbalances in confounding variables between the two groups in that study.

ARDS was associated with higher concentrations of octane in the exhaled breath. This VOC contributed most strongly to the diagnostic model. Octane is an end product of lipid peroxidation, one of the degenerative processes caused by oxidative stress [41, 42]. Oxygen tension is known to influence production of other hydrocarbons (e.g. pentane) but this did not apply to octane in our study as the sensitivity analysis showed that the log odds ratio of the exhaled breath signal did not change when corrected for $\mathrm{IIO}_{2}$. This may be explained by a difference in substrate of the peroxidation process; pentane is the result of peroxidation of $n-6$ polyunsaturated fatty acid (PUFA), linoleic acid or arachidonic acid, while the proposed origin of octane is oleic acid [43]. The latter may not be as dependent on oxygen tension as the former, as suggested by our results. Of note, octane is also present in the breath of healthy individuals, but at very low concentrations [44], and increases due to smoking [45], but this did not influence the diagnostic accuracy in our study.

We also found that higher concentrations of acetaldehyde and 3-methylheptane in the exhaled breath were predictive for ARDS. A variety of bacterial strains can produce acetaldehyde in vitro [16]. Bacterial colonisation of the lung occurs frequently in ventilated ICU patients, and this could have explained the association between ARDS and acetaldehyde. However, the findings that the sensitivity analysis showed no effects of airway colonisation on the log odds ratio for the exhaled breath signal and that patients with unilateral pneumonia included in this study were classified as not having ARDS suggest that bacterial metabolism is not a likely cause for the increased acetaldehyde concentration observed in patients with ARDS in this cohort. Acetaldehyde is also a product of leukocytes [46], and as neutrophil infiltration is a hallmark of ARDS [47], this may be a more appropriate explanation of the difference between patients with ARDS and those without. Branched hydrocarbons like 3-methylheptane were suspected to be produced through lipid peroxidation, similar to octane. However, alkenes and alkynes cannot result from lipid peroxidation because there are no branched PUFAs in the body [43]. To our knowledge, there have not been any reports on an alternative pathway for branched hydrocarbon production despite their association with many oxidative diseases, such as lung cancer [48]. This finding illustrates the hypothesis-generating 
aspect of metabolomics and deserves exploration. By and large, the exhaled breath signal appears to be mainly influenced by (pulmonary) oxidative stress and is further balanced by reflection of inflammatory or infectious processes and an as yet unidentified pathway. Their strength in assessing ARDS appears to be their combination, which should be reflective of their partly independent metabolic pathways.

Our findings show that exhaled breath analysis provides information on the presence or absence of ARDS and this is complementary to pre-test risk assessment by the LIPS. This may not be unexpected as the LIPS consists of risk factors, whereas exhaled breath analysis is based on biochemical alterations that occur during ARDS. Hence, a patient can have a very high a priori risk for disease (LIPS), but in the absence of the biochemical presentation we may still conclude the patient probably does not have the condition (yet). In line with that, if a patient exhibits the biochemical profile with a negligible risk for disease, such a patient probably does not have the condition either. Our data provide evidence that the combination of exhaled breath analysis with the LIPS can be highly suitable for identification of patients with ARDS.

Our study may have a number of strengths. First, the complex mixture of exhaled breath was profiled and the features most strongly linked to ARDS were selected, following the latest recommendations for metabolomics [30, 31]. The diagnostic accuracy of these biological markers was externally validated in a newly recruited population. Second, the influence of disease severity, phenotype and potential confounders was investigated thoroughly. Third, exhaled breath was obtained within $24 \mathrm{~h}$ of ICU admission, in the early phase of disease. Finally, we reported these findings strictly following STARD guidelines to allow for optimal assessment of bias (table E1).

Nevertheless, several limitations should also be noted. First, the consensus criteria for ARDS are excellent for epidemiological research but may not entirely cover the underlying pathophysiology as is meant to be identified using biological markers. Tissue histopathology as a gold standard would have been preferable, but understandably, we could not obtain that in this study. Correlation with other markers of ARDS could have added to our understanding, but the design of this study did not allow additional sampling of blood or to perform BAL. Future studies could correlate breath profiles with changes in markers of ARDS. Second, we included only mechanically ventilated patients. Therefore, the generalisability of our results to spontaneously breathing patients remains to be established. Additional studies are also needed to evaluate the clinical application of exhaled breath analysis for biological monitoring of ARDS progression or resolution and to evaluate the influence of previous intubations for mechanical ventilation, like development of lung injury [49] or airway colonisation [50,51]. Third, we found that cardiopulmonary oedema and unilateral pneumonia patients could be distinguished from ARDS patients. However, the number of included patients with these diagnoses was very low. This may be explained by the rapid increase of noninvasive mechanical ventilation as an alternative to intubation in these patients. Therefore, although we know that the exhaled breath signal is different in patients between pneumonia or cardiogenic pulmonary oedema and ARDS, the accuracy of discriminating these patients cannot be quantified precisely.

The prevalence of ARDS was $\sim 40 \%$ in our study, which is surprisingly high compared with previous reports [52]. Several factors contribute to this finding. First, ventilated post-surgical patients are not routinely admitted to the ICU in our hospital and, when they are admitted because of complications, ARDS is more likely to develop. Second, the included population was severely ill, as exemplified by the high APACHE II score and high mortality. Third, we assessed ARDS prospectively, which could have resulted in the diagnosis of ARDS in patients who would have been missed retrospectively.

The present data suggest that exhaled breath analysis may be used to diagnose ARDS. This study represents a required step towards use of exhaled breath analysis in clinical practice [24]. Next, the predictive value of the observed biological markers should be evaluated in a prospective study including patients who are at risk of ARDS, possibly also including spontaneously breathing patients. Subsequently, the diagnostic accuracy of the combination of risk factors and multiple biological markers should be further improved. In the long run, a move from the diagnostic accuracy paradigm towards a test validation paradigm might be justified [53]. This would allow for the comparison of the added value of several index tests, including exhaled breath analysis, in clinical decisions. Finally, a bedside test should be developed specifically for the detection of VOCs, plausibly octane, acetaldehyde and 3-methylheptane, in the exhaled breath of ICU patients. An array of sensors or rapid mass-spectrometric techniques may be used for that [13]. This may also allow for continuous monitoring [54] of the development of lung injury in ventilated patients.

\section{Conclusion}

Exhaled breath analysis showed good diagnostic accuracy for diagnosing ARDS. Discrimination with controls was reproducible and externally validated. The exhaled breath diagnosis of ARDS was not influenced by severity of illness, ventilator settings or comorbidities. Notably, the diagnostic accuracy 
increased when the exhaled breath analysis was combined with the LIPS. These data suggest that exhaled breath analysis qualifies for the diagnostic assessment of ARDS.

\section{Acknowledgements}

The authors wish to thank all researchers involved in the MARS trial for daily assessment of ARDS and pneumonia in all admitted ICU patients.

\section{References}

ARDS Definition Task Force. Acute respiratory distress syndrome: The Berlin definition. JAMA 2012; 307: 2526-2533.

Thille AW, Esteban A, Fernández-Segoviano P, et al. Comparison of the Berlin definition for acute respiratory distress syndrome with autopsy. Am J Respir Crit Care Med 2013; 187: 761-767.

3 Ferguson N, Meade M, Hallett D, et al. High values of the pulmonary artery wedge pressure in patients with acute lung injury and acute respiratory distress syndrome. Intensive Care Med 2002; 28: 1073-1077.

4 Thompson BT, Matthay MA. The Berlin definition of ARDS versus pathological evidence of diffuse alveolar damage. Am J Respir Crit Care Med 2013; 187: 675-677.

D’Agostino RB. Risk prediction and finding new independent prognostic factors. J Hypertens 2006; 24: 643-645. Pencina MJ, D'Agostino RB, Vasan RS. Evaluating the added predictive ability of a new marker: from area under the ROC curve to reclassification and beyond. Stat Med 2008; 27: 157-172.

7 Gajic O, Dabbagh O, Park PK, et al. Early identification of patients at risk of acute lung injury: evaluation of lung injury prediction score in a multicenter cohort study. Am J Respir Crit Care Med 2011; 183: 462-470.

8 Trillo-Alvarez C, Cartin-Ceba R, Kor DJ, et al. Acute lung injury prediction score: derivation and validation in a population-based sample. Eur Respir J 2011; 37: 604-609.

9 Agrawal A, Matthay MA, Kangelaris KN, et al. Plasma angiopoietin-2 predicts the onset of acute lung injury in critically ill patients. Am J Respir Crit Care Med 2013; 187: 736-742.

10 Nicholson JK, Lindon JC. Systems biology: metabonomics. Nature 2008; 455: 1054-1056.

11 Serkova NJ, Standiford TJ, Stringer KA. The emerging field of quantitative blood metabolomics for biomarker discovery in critical illnesses. Am J Respir Crit Care Med 2011; 184: 647-655.

12 Kiehntopf M, Nin N, Bauer M. Metabolism, metabolome, and metabolomics in intensive care: is it time to move beyond monitoring of glucose and lactate? Am J Respir Crit Care Med 2013; 187: 906-907.

13 Bos LDJ, Sterk PJ, Schultz MJ. Metabolomics in critically ill patients: focus on exhaled air. In: Vincent JL, ed. Annual Update in Intensive Care and Emergency Medicine 2012. Heidelberg, Springer, 2012; pp. 53-64.

14 Pauling L, Robinson AB, Teranishi R, et al. Quantitative analysis of urine vapor and breath by gas-liquid partition chromatography. Proc Natl Acad Sci USA 1971; 68: 2374-2376.

15 Bos LDJ, Sterk PJ, Schultz MJ. Measuring metabolomics in acute lung injury: choosing the correct compartment? Am J Respir Crit Care Med 2012; 185: 789.

16 Bos LDJ, Sterk PJ, Schultz MJ. Volatile metabolites of pathogens: a systematic review. PLoS Pathog 2013; 9: e1003311.

17 Bos LDJ, van Walree IC, Kolk AHJ, et al. Alterations of exhaled breath metabolite-mixtures in two rat models of lipopolysaccharide-induced lung injury. J Appl Physiol (1985) 2013; 115: 1487-1495.

18 Baldwin SR, Simon RH, Grum CM, et al. Oxidant activity in expired breath of patients with adult respiratory distress syndrome. Lancet 1986; 1: 11-14

19 Schubert JK, Muller WP, Benzing A, et al. Application of a new method for analysis of exhaled gas in critically ill patients. Intensive Care Med 1998; 24: 415-421.

20 Fens N, de Nijs SB, Peters S, et al. Exhaled air molecular profiling in relation to inflammatory subtype and activity in COPD. Eur Respir J 2011; 38: 1301-1309.

21 Filipiak W, Sponring A, Bauer M, et al. Molecular analysis of volatile metabolites released specifically by Staphylococcus aureus and Pseudomonas aeruginosa. BMC Microbiol 2012; 12: 113.

22 Guaman AV, Carreras A, Calvo D, et al. Rapid detection of sepsis in rats through volatile organic compounds in breath. J Chromatogr B Analyt Technol Biomed Life Sci 2012; 881-882: 76-82.

23 Knottnerus JA, Muris JW. Assessment of the accuracy of diagnostic tests: the cross-sectional study. J Clin Epidemiol 2003; 56: 1118-1128.

24 Bossuyt PM, Reitsma JB, Bruns DE, et al. Towards complete and accurate reporting of studies of diagnostic accuracy: the STARD initiative. BMJ 2003; 326: 41-44.

25 Moons KGM, Kengne AP, Grobbee DE, et al. Risk prediction models: II. External validation, model updating, and impact assessment. Heart 2012; 98: 691-698.

26 Bernard GR, Artigas A, Brigham KL, et al. Report of the American-European consensus conference on ARDS: definitions, mechanisms, relevant outcomes and clinical trial coordination. Intensive Care Med 1994; 20: 225-232.

27 Klein Klouwenberg PMC, Ong DSY, Bos LDJ, et al. Interobserver agreement of Centers for Disease Control and Prevention criteria for classifying infections in critically ill patients. Crit Care Med 2013; 41: 2373-2378.

28 Horan TC, Andrus M, Dudeck MA. CDC/NHSN surveillance definition of health care-associated infection and criteria for specific types of infections in the acute care setting. Am J Infect Control 2008; 36: 309-332.

29 Bos LDJ, Wang Y, Weda $\mathrm{H}$, et al. A simple breath sampling method in intubated and mechanically ventilated critically ill patients. Respir Physiol Neurobiol 2013; 191: 67-74.

30 Xia J, Broadhurst DI, Wilson M, et al. Translational biomarker discovery in clinical metabolomics: an introductory tutorial. Metabolomics 2013; 9: 280-299.

31 Broadhurst D, Kell DB. Statistical strategies for avoiding false discoveries in metabolomics and related experiments. Metabolomics 2006; 2: 171-196.

32 Chun $\mathrm{H}$, Keles S. Sparse partial least squares regression for simultaneous dimension reduction and variable selection. I R Stat Soc Series B Stat Methodol 2010; 72: 3-25.

33 Moons KGM, Kengne AP, Woodward M, et al. Risk prediction models: I. Development, internal validation, and assessing the incremental value of a new (bio)marker. Heart 2012; 98: 683-690.

34 Flahault A, Cadilhac M, Thomas G. Sample size calculation should be performed for design accuracy in diagnostic test studies. J Clin Epidemiol 2005; 58: 859-862. 

operating characteristic curves: a nonparametric approach. Biometrics 1988; 44: 837-845. injury. J Intensive Care Med 2009; 24: 151-167.

37 Stringer KA, Serkova NJ, Karnovsky A, et al. Metabolic consequences of sepsis-induced acute lung injury revealed by plasma ${ }^{1} \mathrm{H}$-nuclear magnetic resonance quantitative metabolomics and computational analysis. Am J Physiol Lung Cell Mol Physiol 2011; 300: L4-L11.

38 Serkova NJ, Van Rheen Z, Tobias M, et al. Utility of magnetic resonance imaging and nuclear magnetic resonancebased metabolomics for quantification of inflammatory lung injury. Am J Physiol Lung Cell Mol Physiol 2008; 295 : L152-L161.

39 Izquierdo-Garcia JL, Nin N, Ruiz-Cabello J, et al. A metabolomic approach for diagnosis of experimental sepsis. Intensive Care Med 2011; 37: 2023-2032.

40 King J, Mochalski P, Unterkofler K, et al. Breath isoprene: muscle dystrophy patients support the concept of a pool of isoprene in the periphery of the human body. Biochem Biophys Res Comm 2012; 423: 526-530.

41 Riely CA, Cohen G, Lieberman M. Ethane evolution: a new index of lipid peroxidation. Science 1974; 183: $208-210$.

42 Horvat RJ, Lane WG, Ng H, et al. Saturated hydrocarbons from autoxidizing methyl linoleate. Nature 1964; 203: 523-524.

43 Frank Kneepkens CM, Lepage G, Roy CC. The potential of the hydrocarbon breath test as a measure of lipid peroxidation. Free Radical Biol Med 1994; 17: 127-160.

44 Mochalski P, King J, Klieber M, et al. Blood and breath levels of selected volatile organic compounds in healthy volunteers. Analyst 2013; 138: 2134-2145.

45 Wallace LA, Pellizzari ED. Personal air exposures and breath concentrations of benzene and other volatile hydrocarbons for smokers and nonsmokers. Toxicol Lett 1987; 35: 113-116.

46 Shin HW, Umber BJ, Meinardi S, et al. Acetaldehyde and hexanaldehyde from cultured white cells. J Transl Med 2009; 7: 31.

47 Schutte H, Lohmeyer J, Rosseau S, et al. Bronchoalveolar and systemic cytokine profiles in patients with ARDS, severe pneumonia and cardiogenic pulmonary oedema. Eur Respir J 1996; 9: 1858-1867.

48 Gaspar EM, Lucena AF, Duro da Costa J, et al. Organic metabolites in exhaled human breath-a multivariate approach for identification of biomarkers in lung disorders. J Chromatogr A 2009; 1216: 2749-2756.

49 Slutsky AS. Lung injury caused by mechanical ventilation. Chest 1999; 116: 9S-15S.

50 Baram D, Hulse G, Palmer LB. Stable patients receiving prolonged mechanical ventilation have a high alveolar burden of bacteria. Chest 2005; 127: 1353-1357.

51 Craven DE, Hjalmarson KI. Ventilator-associated tracheobronchitis and pneumonia: thinking outside the box. Clin Infect Dis 2010; 51: Suppl. 1, S59-S66.

52 Villar J, Blanco J, Añón JM, et al. The ALIEN study: incidence and outcome of acute respiratory distress syndrome in the era of lung protective ventilation. Intensive Care Med 2011; 37: 1932-1941.

53 Rutjes AW, Reitsma JB, Coomarasamy A, et al. Evaluation of diagnostic tests when there is no gold standard. A review of methods. Health Technol Assess 2007; 11: 1-51.

54 Dolch ME, Frey L, Hornuss C, et al. Molecular breath-gas analysis by online mass spectrometry in mechanically ventilated patients: a new software-based method of $\mathrm{CO}_{2}$-controlled alveolar gas monitoring. J Breath Res 2008; 2 : 037010 . 